\title{
Flux Pinning in Y- and Ag-Doped $\mathrm{MgB}_{2}$
}

\author{
Jack Dyson1, Daniele Rinaldi¹, Gianni Barucca1, Gianni Albertini ${ }^{*}$, Simone Sprio², \\ Anna Tampieri ${ }^{2}$ \\ ${ }^{1}$ SIMAU, Università Politecnica delle Marche, Ancona, Italy \\ ${ }^{2}$ Institute of Science and Technology for Ceramics, National Research Council, Faenza, Italy \\ Email: ${ }^{*}$ albertdom@vodafone.it
}

Received 24 August 2015; accepted 25 October 2015; published 28 October 2015

Copyright (C) 2015 by authors and Scientific Research Publishing Inc.

This work is licensed under the Creative Commons Attribution International License (CC BY).

http://creativecommons.org/licenses/by/4.0/

(c) (i) Open Access

\section{Abstract}

High temperature superconductor research is presently concentrated upon the flux pinning properties of the Abrikosov lattice of the mixed-mode superconducting phase. The temperature thermal fluctuations, current and magnetic field unpin the flux vortices and so cause electromagnetic resistivity in high temperature superconductors. Materials with higher vortex pinning exhibit less resistivity and are more attractive for industrial uses. In the present article, we measured and correlated the pinning flux energy barrier, determined by AC magnetic measurements, and transmission electron microscopy measurements to the critical current $J_{c}$ in Yttrium- and Silver-doped $\mathrm{MgB}_{2}$ superconductors. The energy of the flux vortex was evaluated as a function of the magnetic field. The energy barrier curves suggest an optimal doping level to occur in doped materials. This result only depends on the optimal size and distribution of precipitates, and not on their chemical composition. The energy barriers have been compared with that of undoped $\mathrm{MgB}_{2}$ in literature.

\section{Keywords}

Flux Pinning, AC Magnetic Measurements, Critical Current, Yttrium-Doping, Silver-Doping, $\mathbf{M g B}_{2}$ Superconductors, Transmission Electron Microscopy, X-Ray Diffraction

\section{Introduction}

Magnesium diboride $\mathrm{MgB}_{2}$ BCS-type superconductors (SCs) are widely used in sensors, electric power applications and electronic devices. In fact, even though their critical temperature $T_{c} \sim 39 \mathrm{~K}$ is lower than in traditional cuprate SCs, their upper critical field $H_{c 2}$ is actually higher while an operating temperature of $T \sim 20-30 \mathrm{~K}$ is relatively easy to reach using liquid hydrogen or cryocoolers. The absence of weak links in the matrix (no inter-granular obstacles impede current flow) as well as a strong coupling between grains makes

\footnotetext{
${ }^{*}$ Corresponding author.
}

How to cite this paper: Dyson, J., Rinaldi, D., Barucca, G., Albertini, G., Sprio, S. and Tampieri, A. (2015) Flux Pinning in Yand Ag-Doped $\mathrm{MgB}_{2}$. Advances in Materials Physics and Chemistry, 5, 426-438. 
$\mathrm{MgB}_{2}$ very useful in technological applications [1]. The possible enhancement of the critical temperature and the improvement of the existing high levels of transport current are therefore important topics for this material. On the other hand, the low density of the pure compound and the relative scarcity of pinning centres, actually reduce the sustainable current density in high magnetic fields.

The metal-like structure of $\mathrm{MgB}_{2}$ at room temperature makes it ideal for applications in engineering contexts unlike the cuprate SCs, which exhibits a non-metallic behaviour at room temperature. The latter fact limits the technological applications of cuprate due to difficulties associated with the material processing. As an example, $\mathrm{MgB}_{2}$ can be readily transformed into wires and tapes whereas the well-known cuprate YBCO needs to be deposited onto wires through specifically settled processes.

Superconductivity in $\mathrm{MgB}_{2}$ is based on two differing mechanisms due to its electronic structure. $\mathrm{MgB}_{2}$ has strong $\sigma$ bonds in the B planes and weak $\pi$ bonds in between them. Phonon modes in the Boron planes have a dominant effect due to strong electron-phonon $\left(e^{-}-\phi\right)$ coupling [2] [3], which results in the formation of strong electron pairs confined to the planes. It is related to a complicated Fermi surface, with two superconducting bands, which induces a strongly anisotropic conductivity in the lattice structure. The two bands produce different levels of superconductivity, the intra-planar variant being the weaker, leaving a particularly large conductivity in the Boron $a b$-plane. The high critical temperature $T_{c}$ of $\mathrm{MgB}_{2}$ is due to the electron-phonon coupling in the plane [4] where the inter- and intra-band scattering effects tend to reduce the $T_{c}$ value [4] [5].

The complicated dual band superconductivity structure of $\mathrm{MgB}_{2}$ affects the main superconducting parameters like the irreversibility field, $H_{i r r}$, the upper critical field $H_{c 2}$, the critical superconducting temperature $T_{c}$ and, importantly, also the critical current $J_{c}$ [6] [7]. In order to increase the values of the last two parameters, the introduction of controlled amounts of doping agents is an interesting procedure: in past experiments, the critical superconducting field was observed to rise but the critical temperature remained idle [7]. To the best of our knowledge, increase of $T_{c}$ induced by doping $\mathrm{MgB}_{2}$ was only reported in the high field case of nano-C doped $\mathrm{MgB}_{2}$ [8].

Conversely, it was found that the critical current could be modified through doping. Indeed, in $\mathrm{MgB}_{2}$ the current flow is not governed by the weak link mechanism and hence the vortex dynamics alone determines the value of $J_{c}$. Compounds like $\mathrm{Nb}_{3} \mathrm{Sn}$ support a higher $J_{c}$ [9] because factors like mass density, packing fraction and connectivity as well as impurities like MgO play a major enhancing role in the material. In type II SCs, operating in the mixed regime, the Abrikosov flux lattice can be driven by the electric field, which induces a backward electromotance, dissipating power as the flux changes through the circuit. In such complicated scenarios, thermally activated flux flow (TAFF) and flux creep have been observed [10]. The remedy to this is the introduction of pinning centres locking the flux vortices with a potential thus allowing the critical current to increase. Different types of pinning centres can be introduced, e.g. grain boundaries, point defects as well as impurities and lattice variations brought on by doping [8].

In order to make the formation of pinning centres effective to increase $J_{c}$, they should exhibit sizes as large as the coherence length [8]. In $\mathrm{MgB}_{2}$ it is valued in the range between 2 and $10 \mathrm{~nm}$ [8] [11]. Zehetmayer et al. [7] report that neutron radiation induces defects of about 5 - $10 \mathrm{~nm}$ in size, thus creating pinning centres that influence mainly the intra-band $e^{-}-\phi$ scattering. Compared to the alternative Carbon doping, neutron irradiation seems an efficient method for adding pinning centres into the material. Since grain boundaries also act as pinning centres, the decreasing grain dimensions improve the critical current. Beside the pinning centres, the densification of the compound in general enhances the superconducting state.

For $\mathrm{MgB}_{2}$ in particular, good results in $J_{c}$ enhancement have been obtained by the use of doping agents. Different elements have been used so far, spanning from rare-earths to magnetic elements as well as Silicon [5] [12] [13]. The best results are obtained when Carbon substitutes Boron. Carbon is an electron donor that can influence the Fermi surface of the $\mathrm{MgB}_{2}$ lattice. The result is a significant enhancement of $H_{c 2}$ and $J_{c}$ through enhanced intra-band scattering [7]. The Carbon substitution in the Boron layer causes an in-plane lattice distortion, tending to reduce the coherence length. $H_{i r r}$ and $H_{c 2}$ actually increase owing to the extra intraband scattering in $\sigma$ and $\pi$ orbitals. This improves the flux pinning in high fields and in turn $J_{c}$ when the doping levels are optimal [11] [14]. Unfortunately, these gains were accompanied by a lowering of $T_{c}$.

Alternatively, SiC doping causes lattice distortion as well dislocation, which in turn acts as pinning centres [15] [16]. Good results have been obtained also by means of graphene doping [16] [17].

Improvement of $J_{c}$ can also occur without ion substitution in the $\mathrm{MgB}_{2}$ lattice. In this respect, $J_{c}$ values higher than in pure sample were found in Cu-doped samples, due to the formation of an additional $\mathrm{MgCu}_{2}$ phase 
that acted as pinning centres [18] [19]. If, on the contrary, substitution of Magnesium with Copper occurs, a hole-donor compound is obtained, which produced an unexpected reduction in $T_{c}$ [20] [21]. Also Silver doping in $\mathrm{MgB}_{2}$ yielded an increase of the critical current, due to the formation of MgAg nanoparticles that acted as pinning centres [22]-[24].

On the basis of these previous investigations, it can be concluded that, since $J_{c}$ depends on the vortex dynamics, a high flux pinning efficiency is fundamental in sustaining high levels of critical current.

This paper studies the behaviour of the vortex pinning potential (barrier) in Magnesium diboride doped with Silver or Yttrium, a hole and an electron donor, respectively. Samples are prepared by means of hot isostatic pressing (HIP) or uniaxial hot pressing (UHP) techniques, respectively. The correlation between structural properties and performance, in terms of critical current, was analysed in order to elucidate the importance of pinning centres in doped $\mathrm{MgB}_{2}$. In particular, scope of this work is to clarify the influence of Silver and Yttrium doping agents (at our best knowledge the latter was rarely studied) on the height of the vortex energy barrier. This last is a fundamental property for improving the superconducting critical current in the presence of external magnetic fields. Moreover, the model used to evaluate the flux energy barrier proved to be a convenient tool for the analysis and the comprehension of the $J_{c}$ magnitude in relation with structural parameters. In fact, non linear and complex phenomena involved in the depinning mechanism need special care in the data analysis.

In a previous study of $\mathrm{MgB}_{2}$ [23], it was discussed that the sample purity and density were not directly related to the superconducting properties, while the pinning centres were suggested to play a fundamental role. Interesting results in this sense were obtained with small amounts of Ag and Y doping. In order to make this last point clear, our results are compared with those reported in literature in the case of undoped $\mathrm{MgB}_{2}$ samples, either synthesized as bulk or as film.

\section{Experimental}

UHP (Y-doped) and HIP (Ag-doped) techniques have been used to obtain consolidated $\mathrm{MgB}_{2}$ bulk samples. The UHP (Uniaxial Hot Pressing) samples were prepared as follow, magnesium, boron and yttrium powders in the composition $\operatorname{Mg}_{1-\mathrm{x}} \mathrm{Y}_{\mathrm{x}} \mathrm{B}_{2}$, have been heated in vacuum at a rate of $\sim 25 \mathrm{~K} / \mathrm{min}$ up to the sintering temperature of $1373 \mathrm{~K}$. At $T=773 \mathrm{~K}$, a pressure of $30 \mathrm{MPa}$ was applied and kept constant. The sample was kept at the final temperature for $20 \mathrm{~min}$ and then spontaneously cooled up to ambient temperature under vacuum. No pressure is applied during the cooling. The HIP (Hot Isostatic Pressing) samples were prepared starting from the composition $\mathrm{Mg}_{1-\mathrm{x}} \mathrm{Ag}_{\mathrm{x}} \mathrm{B}_{2}$. At first, a pressure of $150 \mathrm{MPa}$ in inert atmosphere was applied, followed by a cold isostatic pressing at $300 \mathrm{MPa}$. The pellets were then encapsulated under vacuum prior pressure-heating treatments. Pressure and temperature were increased to reach the final value simultaneously (150 MPa and $1073 \mathrm{~K}$, for 1 hour). Then the samples spontaneously cool under high pressure until the temperature of $373 \mathrm{~K}$, then the pressure was lowered up to atmospheric pressure.

These complex preparation techniques are explained in detail in Ref. [23]. The samples are listed in Table 1. Once manufactured, the samples were structurally characterized by X-ray diffraction (XRD) using a Bruker D8 Advance diffractometer (operating with a $\mathrm{Cu}-\mathrm{K} \alpha$ radiation source) together with transmission electron microscopy (TEM), carried out on a Philips CM200 electron microscope operating at $200 \mathrm{kV}$. For TEM observations, the samples were prepared by scraping small amounts of powder from each pellet over carbon coated copper grids.

Table 1. Studied samples with molar composition and preparation technique.

\begin{tabular}{cc}
\hline Sample & Technique \\
\hline $\mathrm{AG} 1: 1 \mathrm{Mg}+2 \mathrm{~B}+0.01 \mathrm{Ag}$ & $\mathrm{HIP}$ \\
$\mathrm{AG} 5: 1 \mathrm{Mg}+2 \mathrm{~B}+0.05 \mathrm{Ag}$ & $\mathrm{HIP}$ \\
$\mathrm{AG} 20: 1 \mathrm{Mg}+2 \mathrm{~B}+0.2 \mathrm{Ag}$ & $\mathrm{HIP}$ \\
$\mathrm{Y} 05: 1 \mathrm{Mg}+2 \mathrm{~B}+0.005 \mathrm{Y}$ & $\mathrm{UHP}$ \\
$\mathrm{Y} 10: 1 \mathrm{Mg}+2 \mathrm{~B}+0.010 \mathrm{Y}$ & $\mathrm{UHP}$ \\
$\mathrm{Y} 15: 1 \mathrm{Mg}+2 \mathrm{~B}+0.015 \mathrm{Y}$ & UHP \\
\hline
\end{tabular}


The AC magnetic measurements were carried out on a Quantum Design PPMS system. Complex magnetic susceptibility data were collected as a function of temperature by applying a static field (varying from 1.5 to 7.0 $\mathrm{T}$ ), being the AC field fixed at 1 Oersted, with frequency (f) varying from $100 \mathrm{~Hz}$ to $10 \mathrm{KHz}$ for each applied static field. The nonlinear complex susceptibility has been measured up to the third harmonic for each measuring frequency. These experimental measurements are fitted using nonlinear numerical analysis (see appendix) and values for the flux pinning potential (energy barrier) calculated in each case [22] [25]-[28]. These were grouped according to the applied field for each case of $\mathrm{Y}$ and Ag doping.

\section{Results and Discussion}

X-ray patterns recorded for the Ag- and $\mathrm{Y}$-doped $\mathrm{MgB}_{2}$ samples are reported in Figure 1. In the whole range of investigation, no significant change of $\mathrm{MgB}_{2}$ lattice parameters was found, within experimental error. Thus we concluded that no substitutions occurred in the $\mathrm{MgB}_{2}$ unit cell.

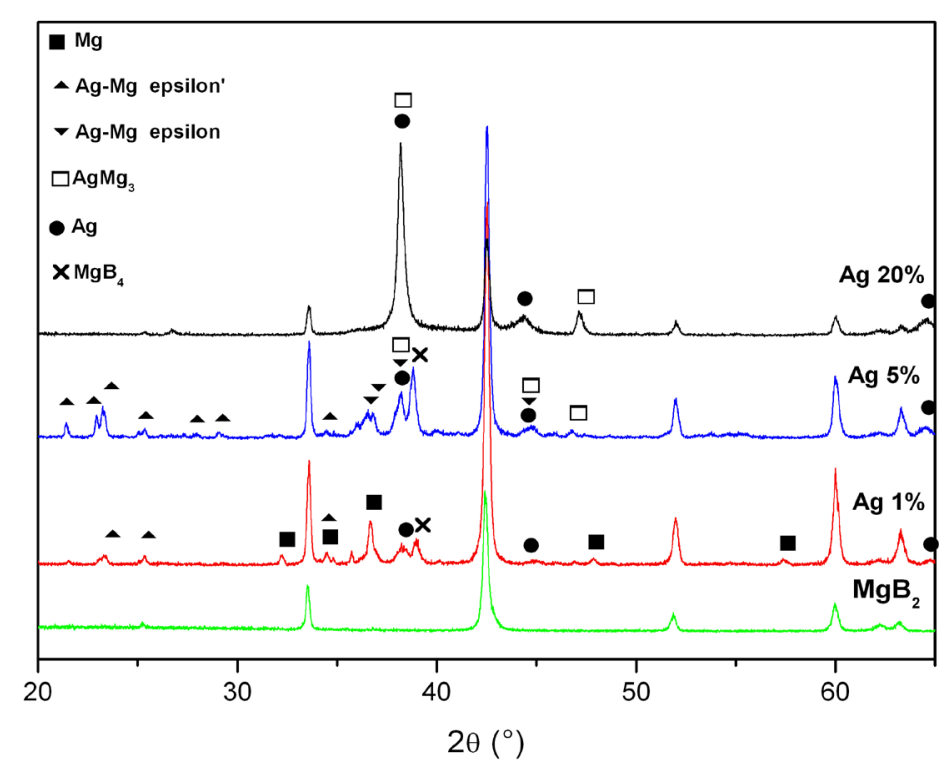

(a)

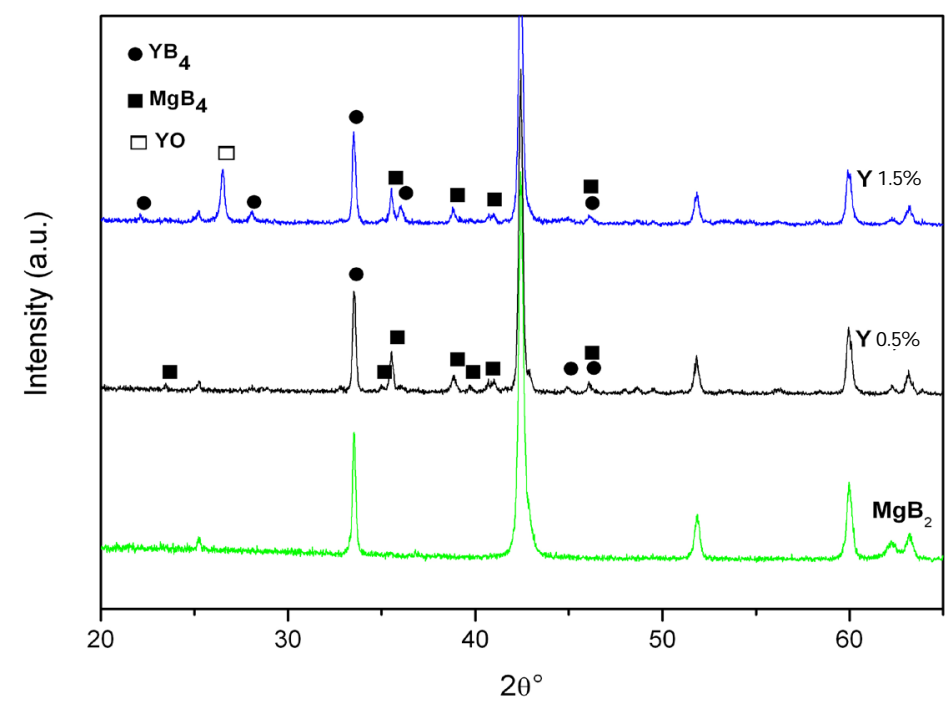

(b)

Figure 1. X-ray patterns of Ag-doped (a) and Y-doped (b) $\mathrm{MgB}_{2}$ samples. A reference scan from an undoped $\mathrm{MgB}_{2}$ sample (prepared by HIP) is also reported. 
All the diffraction peaks of the undoped sample are attributed to pure $\mathrm{MgB}_{2}$ and $\mathrm{MgO}$ compounds while additional peaks are observed in the doped samples.

For the Ag-doped samples, the increase in Ag concentration caused the Mg peaks to disappear while the phase Ag-Mg, presents as $\varepsilon^{\prime}-\mathrm{Ag}_{0.24} \mathrm{Mg}_{0.76}$ (ICDD card number 39-0992) in the AG1 sample, as $\varepsilon^{\prime}-\mathrm{Ag}_{0.24} \mathrm{Mg}_{0.76}$ and $\varepsilon-\mathrm{AgMg}_{3}$ (ICDD card number 39-0991) in AG5, becomes $\mathrm{AgMg}_{3}$ (ICDD card number 01-1170) in the AG20 sample. Furthermore, the presence of metallic Ag was found to increase with the amount of Ag introduced in the $\mathrm{MgB}_{2}$ matrix. The mean dimension of the Ag-Mg grains evaluated through the Scherrer formula was $9 \pm 1 \mathrm{~nm}$ for the AG1 sample, $10 \pm 1 \mathrm{~nm}$ for AG5 and $13 \pm 1 \mathrm{~nm}$ for AG20.

In the Y-doped samples, $\mathrm{X}$-ray measurements showed the presence of $\mathrm{MgB}_{4}$ (ICDD card number 73-1014) and $\mathrm{YB}_{4}$ (ICDD card number 79-1992) phases in addition to $\mathrm{MgB}_{2}$ and $\mathrm{MgO}$. The intensity of $\mathrm{MgB}_{4} \mathrm{Peaks}$ remained unchanged with the Yttrium content, whereas those belonging to $\mathrm{YB}_{4}$ increased. The evaluated average grain size of $\mathrm{MgB}_{4}$ was the same for both Y05 and Y15 samples: $d=54 \pm 1 \mathrm{~nm}$. The average grain size of $\mathrm{YB}_{4}$ was $13 \pm 3 \mathrm{~nm}$ for $\mathrm{Y} 05$ and $38 \pm 2 \mathrm{~nm}$ for $\mathrm{Y} 15$.

Better results in XRD analysis with respect to a previous work [23] are due to the improved instrument resolution. The sample Y10 could not be analysed by XRD but this deficiency does not invalidate the conclusions of the present work, as highlighted in the following discussion.

Transmission Electron Microscopy (TEM) analyses of Ag- and Y-doped samples confirmed the results of $\mathrm{X}$-ray analysis and show the presence of precipitates outside the $\mathrm{MgB}_{2}$ grains. In particular two bright field images for sample AG5 are given in Figure 2. Small dark precipitates of size ranging from $10 \mathrm{~nm}$ to $20 \mathrm{~nm}$ are also easily visible. The corresponding electron diffraction patterns are shown in the insets. Diffraction spots and rings can be attributed to the presence of the $\mathrm{MgB}_{2}$ lattice and Magnesium-Silver phases (it was not actually possible to distinguish between $\mathrm{Ag}$ or $\mathrm{Mg}_{3-\mathrm{x}} \mathrm{Ag}_{\mathrm{x}}$ by TEM diffraction analyses).

Figure 3 shows two bright field images of the Y-doped sample Y15. Small dark precipitates having sizes ranging from few nanometres to a few tens of nanometres are visible. The diffraction images related to these areas indicate the presence of $\mathrm{MgB}_{4}$ and $\mathrm{YB}_{4}$ phases (it was not possible to distinguish between the two phases).

The values of $J_{c}$ and $T_{c}$ were measured through the AC complex magnetic susceptibility [23]. From these parameters the ability of the material to support the superconducting state can be inferred. A measure of the quality of a superconductor is the transition ratio, TR (defined as $\Delta T_{T R} / T_{c}$ where $\Delta T_{T R}$ is the transition width, for more details see Ref. [21]). A further parameter is the ratio $\Delta T_{200} / T_{c}$, with $\Delta T_{200}=T_{C}-\left.T\right|_{J_{c}=200 \mathrm{~A} / \mathrm{cm}^{2}}$.

According to Table 2, the Ag- and Y-doped samples have relatively good performances as superconductors

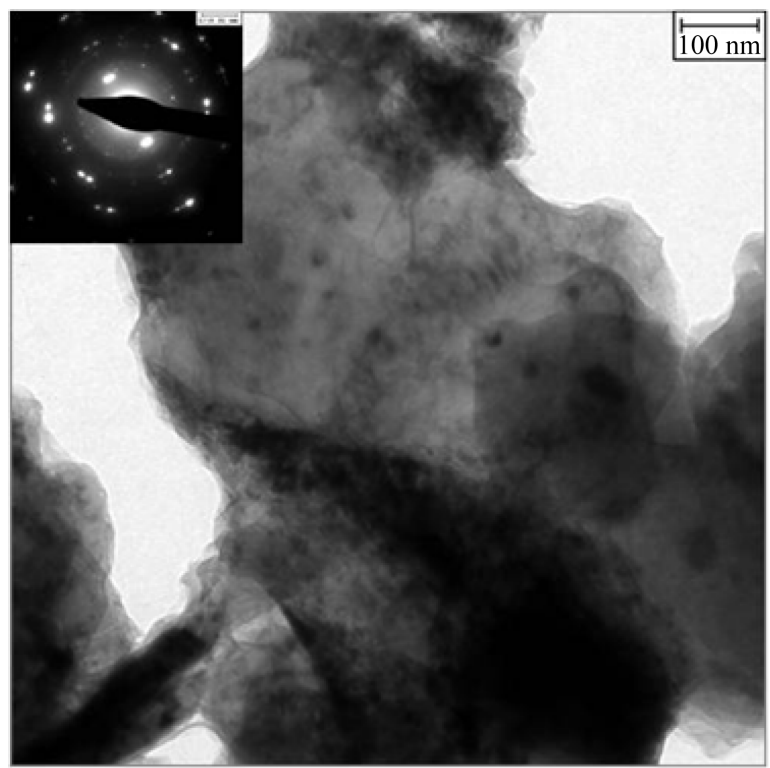

(a)

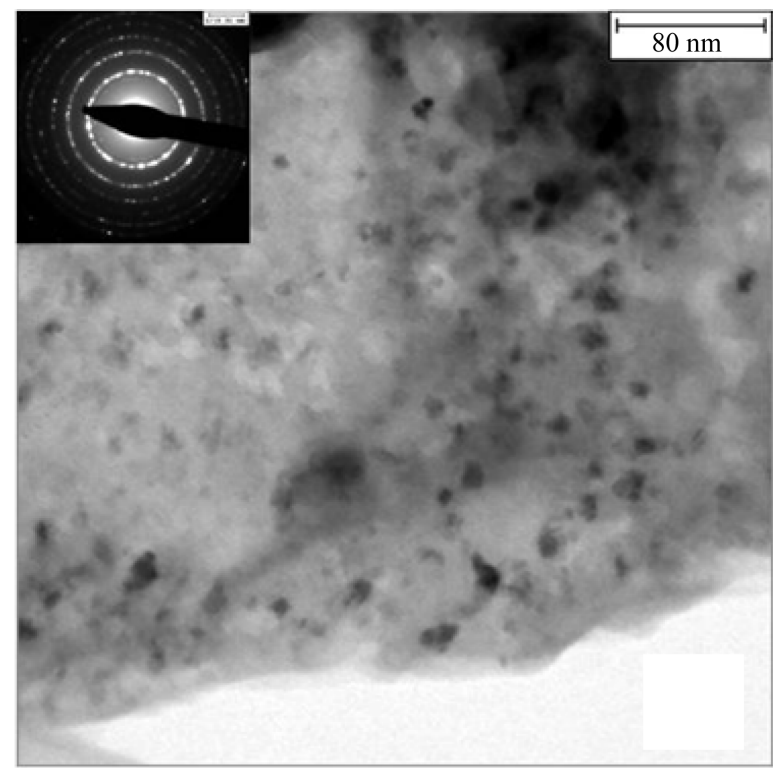

(b)

Figure 2. (a) Sample AG5 seen as a TEM image and as a diffraction pattern (inset). (b) Sample AG5 seen as a TEM image and as a diffraction pattern (inset) at around twice the magnification of image (a). 


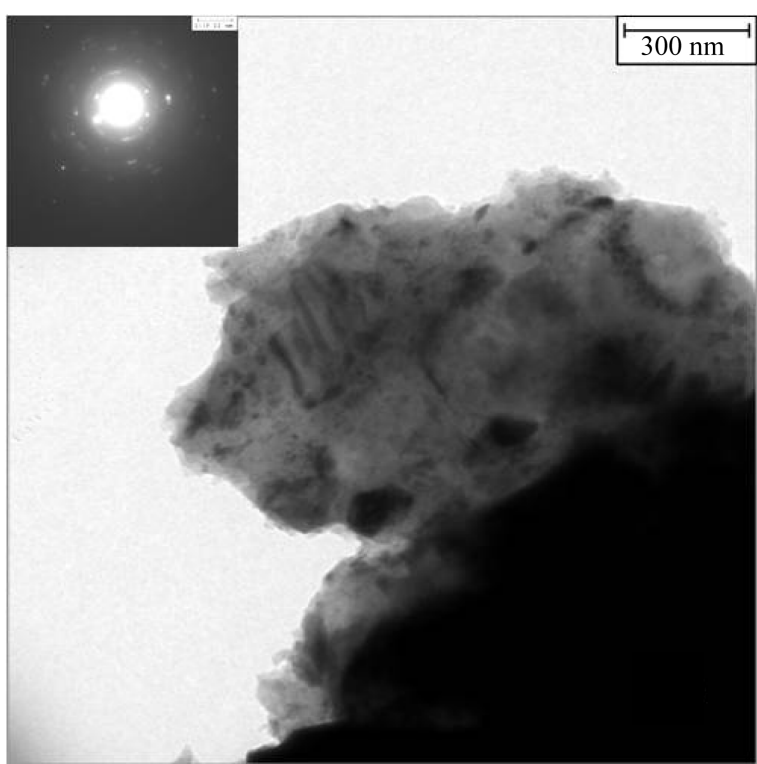

(a)

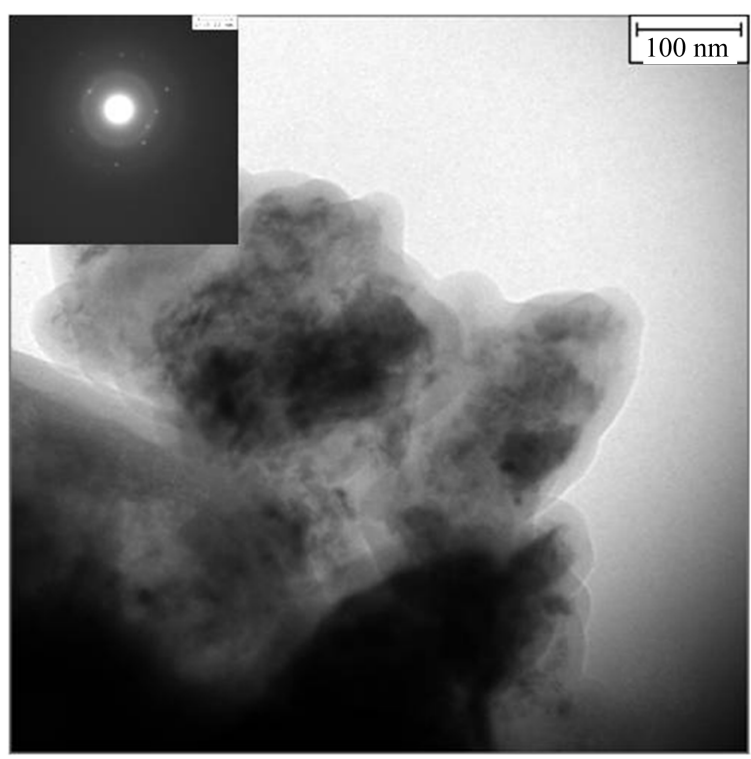

(b)

Figure 3. (a) Sample Y15 seen as a TEM image and as a diffraction pattern (inset); (b) Sample Y15 seen as a TEM image and as a diffraction pattern (inset) at around thrice the magnification of image (a).

Table 2. Superconductor behaviour parameterised according to the $T_{c}, T R$ and $\Delta T_{200}$ indices.

\begin{tabular}{cccc}
\hline Sample & $T_{c}(K) \mathbf{( \pm \mathbf { 0 . 2 5 } \% )}$ & $T R \mathbf{( \pm \mathbf { 8 } \% )}$ & $\frac{\Delta T_{200}}{T_{c}}(\times 100)( \pm \mathbf{8} \%)$ \\
AG1 & 38.45 & 0.0036 & 0.62 \\
AG5 & 38.53 & 0.0057 & 0.70 \\
AG20 & 38.38 & 0.0078 & 1.04 \\
Y05 & 38.85 & 0.0154 & 0.59 \\
Y10 & 38.85 & 0.0129 & 0.72 \\
Y15 & 38.81 & 0.0173 & 0.67 \\
\hline
\end{tabular}

and the two groups show similar behaviour albeit at different levels. In particular, the critical temperature is comparable to those reported in literature for $\mathrm{MgB}_{2}$ superconductors.

The critical current was valued by means of AC magnetic susceptibility as a function of temperature in a range just below the critical temperature in absence of a DC applied field. Overall trends in $J_{c}$ for various samples are shown in Figure 4. In terms of the absolute temperature, the Y-doped group obtained better performance. No major difference in absolute current has been detected among the Y-doped samples but degradation is evident in the AG20 sample among the Ag-doped samples.

For a deeper understanding of the critical current behaviour, we consider the pinning mechanism in the superconducting regime and compute the energy barrier for the pinning, especially when an external field is applied. In this framework the higher the flux pinning barrier, the higher the maximum current the sample will support. In fact, when the magnetic flux detaches, the Lorentz forces induce a finite resistivity, which destroys the superconducting state. In this context different models based on the complex magnetic AC susceptibility measurements in presence of applied external fields have been proposed. Zazo et al. valued the activation energy for the flux by applying the Arrhenius law [26]. For a YBaCuO polycrystalline sample, they reported an activation energy varying from 3.41 to $1.41 \mathrm{eV}$; in this respect the Arrhenius law turned out to be too simple to correctly interpret the complex flux dynamics of the system in our case.

In order to improve the analysis, Qin et al. [27] suggested that the dependence of the flux creep activation 


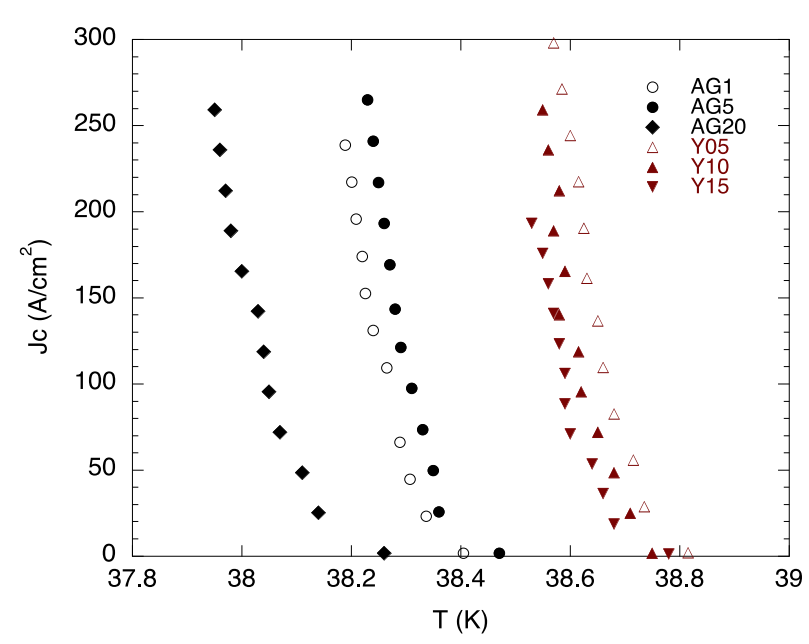

Figure 4. The critical current derived from AC susceptibility measurements plotted as a function of the temperature.

energy upon applied magnetic field follows a power law and proposed the following mathematical model:

$$
\ln f-\ln f_{0}=-\frac{U(T) U_{0}\left(J_{C}, B_{D C}\right)}{k_{B} T}
$$

where $U_{0}$ is the flux pinning energy barrier, $B_{D C}$ is the applied magnetic induction,

$$
J_{c}=\frac{H_{A C}}{d}
$$

is the critical current evaluation according to the Bean critical state model [23] [29], $H_{A C}$ is the measuring field, $d$ is the sample half-thickness, $U(T)$ is a temperature scaling function

$$
U(T)=\left[1-\left(\frac{T}{T_{i r r}}\right)^{2}\right]^{2}
$$

and $T_{\text {irr }}$ is the irreversibility temperature to be determined from the data.

On the left hand side of Equation (1), $f$ is the measurement frequency and $f_{0}$ is the inverse of the characteristic time scale.

Amalgamating these expressions gives the experimental relationship:

$$
y=U_{0}\left(J_{C}, B_{D C}\right) C(T)+\ln f_{0}
$$

where $y=\ln (f)$ and

$$
C(T)=U(T) / k_{B} T .
$$

$T$ is set to $T_{p}$, the temperature at which the maximum in the imaginary part of the complex susceptibility occurs [27]. In fact at this temperature the complete flux penetration is established for every tuple $\left(B_{D C}, f\right)$ [29]. At this point it is sufficient to set the value of $T_{i r r}$ to the onset temperature of third susceptibility harmonic valued at the higher frequency [28]. Equation (4) is linear with respect to $C\left(T_{p}\right)$, plotting it in the $y-C$ plane at constant $B_{D C}$. Unfortunately, using Equation (4), as a model with the present data, does not satisfactorily produce the expected straight line with slope $U_{0}\left(J_{C}, B_{D C}\right)$ and intercept $\ln \left(f_{0}\right)$.

Ghigo et al. [28] suggested the modified equation which we adopt here:

$$
\ln f-\ln f_{0}=-\frac{U_{0}}{k_{B} T}\left[1-\left(\frac{T}{T_{i r r}}\right)^{2}\right]^{\alpha} .
$$

Setting $x=T / T_{\text {irr }}$ and $y=\ln (f)$ we obtain: 


$$
y=-\left(\frac{U_{0}}{k T_{i r r}}\right) \frac{\left[1-x^{2}\right]^{\alpha}}{x}+\ln f_{0} .
$$

The experimental $x$ values are calculated with $T=T_{p}$ as above. The equation above is fitted using a nonlinear algorithm to $(x, y)$ data to get values for $\alpha$ and $U_{0}$ (see Appendix). Applying this model a satisfactory fit is obtained: the graph in Figure 5 is a plot of this fitted curve for different field levels for the $20 \% \mathrm{Ag}$ doping.

In Figure 6 the valued $U_{0}$ are plotted as a function of applied field.

The phenomenological data analysis procedure described above gives the simplest, most reliable impression of how doping influences the energy barrier variation supported by the data.

Both for Yttrium and Silver a decrease of $U_{0}$ barrier is observed at increasing applied magnetic field. At a fixed field, the $U_{0}$ values do not always increase with increasing concentration of dopant, thus indicating that

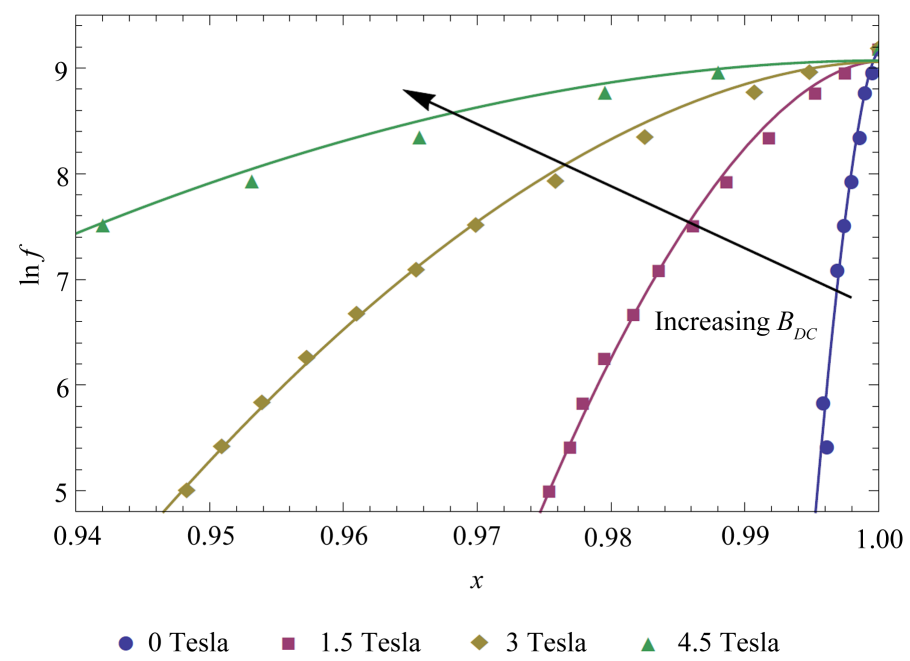

Figure 5. The frequency logarithm $(\ln f)$ of the measurement AC magnetic field plotted against $x$ (as defined in the text). The power law (7) is fitted to $x$ valued from the experimental data. The fit concerns the $20 \%$ Ag doped $\mathrm{MgB}_{2}$ sample at different DC field: $B_{D C}$ field is expressed in Tesla.

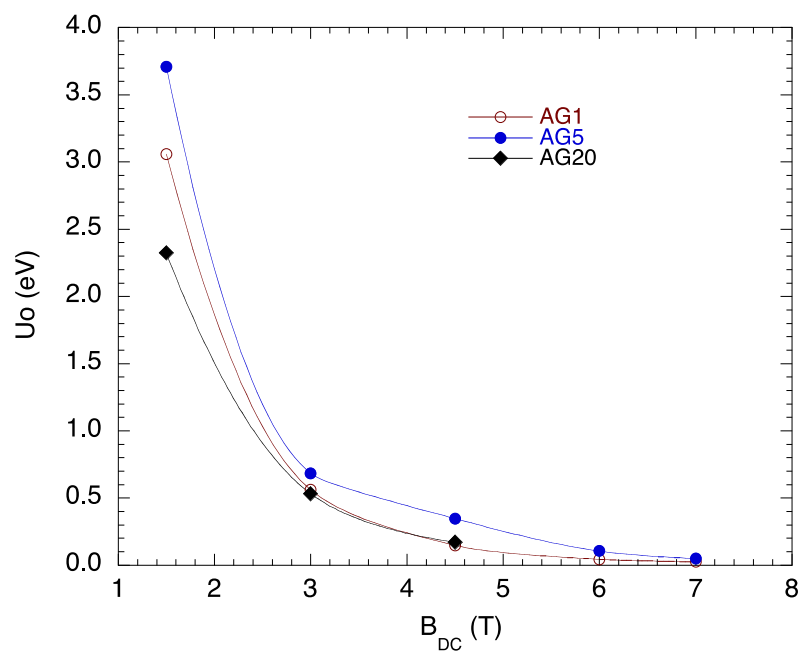

(a)

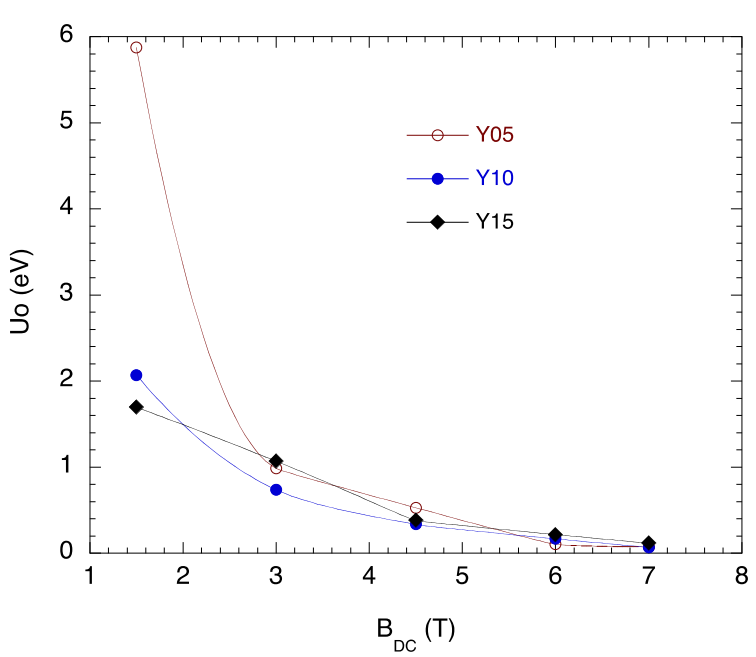

(b)

Figure 6. The energy barrier variation $U_{0}$ with applied DC field across each doping series (the zero field point omitted due to low confidence). (a) Ag series. (b) Y series. 
optimal concentrations for obtaining high barriers occurs.

The data in Figure 6 resemble a power law: however, at high field values the samples exhibit the same behaviour regardless of doping content.

These experiments suggest the occurrence of a maximum doping level in a type II superconductor for the optimal flux pinning properties-a possible consequence of which is the maximum sustainable $J_{c}$ for the material.

No significant improvements have been detected in $T_{c}$ although the doped samples exhibit high $T_{c}$ (ranging from $38.85 \mathrm{~K}$ to $38.38 \mathrm{~K}$ ). As outlined in the introduction, the value of $T_{c}$ depends on the electron-phonon coupling as well as the intra- and inter-band scattering and electron density.

The values of $T_{c}$ and $J_{c}$ could change in principle when Y substitutes for Mg because $\mathrm{Y}$ is an electron donor. In our case, although there is no experimental evidence for Mg substitution in the crystalline structure, the critical current is affected by the introduction of Ag or Y. This can be explained in terms of the formation of pinning centres. In fact both the X-ray and TEM analyses demonstrated the existence of non-superconducting precipitates, with dimensions in the range 2 to $10 \mathrm{~nm}$; exactly those required to act as efficient pinning centres [7] [8]. In the Y-doped samples, among the other precipitates with higher dimensions, the mean radius for the $\mathrm{YB}_{4}$ is $13 \pm 3 \mathrm{~nm}$ for the sample at $0.5 \%$ doping, increasing up to $38 \pm 2 \mathrm{~nm}$ for $1.5 \%$ doping.

In the Ag-doped samples we detected in $\mathrm{MgB}_{2}$ doped at $1 \% \mathrm{Ag}$ nanometric precipitates of $\mathrm{MgO}$ and $\mathrm{Ag}_{\mathrm{x}} \mathrm{Mg}_{3-\mathrm{x}}$. These precipitates, having a mean radius of $9 \pm 1 \mathrm{~nm}$, tend to become larger with Ag concentration $(D=10 \pm 1$ $\mathrm{nm}$ in AG5 and $d=13 \pm 1 \mathrm{~nm}$ in AG20) and moreover will transform into the $\mathrm{AgMg}_{3}$ phase. The precipitate size increases with the dopant concentration for both types of dopant.

The behaviour of the calculated energy barrier is in agreement with the hypothesis concerning the role played by pinning centres. The Y doping is more effective than Ag at very low dopant concentration and low fields. In all cases the pinning barrier decreases rapidly with the field.

The $\mathrm{MgB}_{2}$ doped at $0.5 \% \mathrm{Y}$ has a possible $\mathrm{YO}_{\mathrm{x}}$ phase as well as a better distribution of pinning centres compared with the sample doped at $1.5 \% \mathrm{Y}$ which has larger $\mathrm{YB}_{4}$ precipitates. Both of these contain $\mathrm{MgO}$ and $\mathrm{MgB}_{4}$.

In the case of the Ag-doped samples, increasing concentration of Ag increases the number of precipitates such as Ag-Mg of appropriate size to act as pinning centres. By exceeding a certain value of the concentration of Ag, precipitates tend to grow (in size, but probably also in density) losing their effectiveness as pinning centres. The energy barrier variation for both types of doping follows a similar trend.

The effectiveness of doping to increase the energy barrier is deduced after comparing these experimental data with those obtained from undoped $\mathrm{MgB}_{2}$ samples reported in literature. In $\mathrm{MgB}_{2}$ film [30], $U_{0}$ is about $0.9 \mathrm{eV}$ for magnetic field in the range $0-1 \mathrm{~T}$, decreasing down to 0.08 at about $7 \mathrm{~T}$. The data have been valued by fitting $\rho$ to the Arrhenius law. In bulk $\mathrm{MgB}_{2}$ Jin et al. valued $U_{0}$ ranging from $0.6 \mathrm{eV}$ to $0.25 \mathrm{eV}$ at 0.5 and $1 \mathrm{~T}$ respectively, by means of current measurements [31]. Senatore et al. compared the experimental results of AC measurements in Bulk $\mathrm{MgB}_{2}$, with susceptibility curves obtained by numerical calculations, assuming a nonlinear diffusion equation for the magnetic field. They obtained good results fitting with $U_{0}=0.7 \mathrm{eV}$ at $0.02 \mathrm{~T}$ [32]. Although the reported data are obtained from different samples and different techniques, they confirm that the doping strongly modifies the barrier height. Moreover, at high field (7 - 9 T) Patnaik et al. found $\mathrm{U}_{0}$ about $0.17 \mathrm{eV}$ [33], in textured film which is comparable to the values presented in this work, thus confirming that at high field the studied pinning centres are less effective (see also above and Ref. [30]).

Our data indicate a strong increase of the energy barrier in doped samples with respect to all these values obtained in the undoped samples, at least at low fields (less than about $5 \mathrm{~T}$ ).

The obtained $U_{0}$ values indicate an optimal doping level for both the dopants (about $5 \%$ for $\mathrm{Ag}$ and $0.5 \%$ for Y doping). The dopant excess degrades the samples and introduces disorder.

\section{Conclusions}

The $\mathrm{Y}$ and $\mathrm{Ag}$ doped $\mathrm{MgB}_{2}$ superconductors have been studied by means of magnetic, XRD and TEM measurements. The flux pinning energy barrier $U_{0}$ has been evaluated and compared among the different samples. The energy barrier height $U_{0}$ ranges from 3.5 to $6 \mathrm{eV}$ for the $\mathrm{Ag}$ and $\mathrm{Y}$ doped samples respectively at low field and fast decreases with the applied magnetic field. The $U_{0}$ values of undoped samples shown in the literature (lower then $1 \mathrm{eV}$ at $1 \mathrm{~T}$ ) are lower with respect to the doped samples here reported (higher then about $2 \mathrm{eV}$ at $1 \mathrm{~T}$ ), 
thus putting in evidence the efficiency of doping, at least at low magnetic field (about $5 \mathrm{~T}$ ), as explained in the discussion. Better performance levels were achieved in cases where doping was low. As the doping agents do not substitute $\mathrm{Mg}$, the reported results can be considered a clue that efficiency of the pinning centres depends on their size and distribution. The detection of optimal doping levels can be related to the occurrence of optimal dimensions and distributions of the precipitates.

As Y-doping is rarely studied, this paper also reports useful information on that electron donor dopant.

\section{References}

[1] Chandrashekar, R.G., Tiwari, R.S., Srivastava, O.N. and Malik, S.K. (2007) Enhancement of Flux Pinning and High Critical Current Density in Graphite Doped $\mathrm{MgB}_{2}$ Superconductor. Journal of Applied Physics, 101, Article ID: 043906.

[2] Buzea, C. and Yamashita, T. (2001) Review of the Superconducting Properties of $\mathrm{MgB}_{2}$. Semiconductor Science and Technology, 14, R115. http://dx.doi.org/10.1088/0953-2048/14/11/201

[3] Cheng, C.H., Zhao, Y., Zhu, X.T., Nowotny, J., Sorrell, C.C., Finlayson, T. and Zhang, H. (2003) Chemical Doping Effect on the Crystal Structure and Superconductivity of $\mathrm{MgB}_{2}$. Physica C: Superconductivity, 386, 588-592. http://dx.doi.org/10.1016/S0921-4534(02)02167-6

[4] Cohen, L.F., Bugoslavsky, Y., Perkins, G.K., Moore, J., Miyoshi, Y. and Caplin, A.D. (2004) Magnetic Properties of $\mathrm{MgB}_{2}$ in the Presence of Disorder. Physica C, 408-410, 628-631. http://dx.doi.org/10.1016/j.physc.2004.03.087

[5] Wang, X.-L., Dou, S.X., Hossain, M.S.A., Cheng, Z.X., Liao, X.Z., Ghorbani, S.R., Yao, Q.W., Kim, J.H. and Silver, T. (2010) Enhancement of the In-Field Jc of $\mathrm{MgB}_{2}$ via $\mathrm{SiC}_{14}$ Doping. Physical Review B, 81, Article ID: 224514.

[6] Kim, J.H., Dou, S.X., Oh, S., Jercinovic, M., Babic, E., Nakane, T. and Kumakura, H. (2008) Correlation between Doping Induced Disorder and Superconducting Properties in Carbohydrate Doped $\mathrm{MgB}_{2}$. Journal of Applied Physics, 104, Article ID: 063911. http://dx.doi.org/10.1063/1.2980275

[7] Zehetmayer, M., Krutzler, C., Eisterer, M., Jun, J., Kazakov, S.M., Karpinski, J. and Weber, H.W. (2006) Effect of Disorder on the Irreversible Magnetic Properties of Single Crystalline $\mathrm{MgB}_{2}$ : Comparison of Carbon Doping and Neutron Irradiation. Physica C, 445-448, 65-68. http://dx.doi.org/10.1016/j.physc.2006.03.079

[8] Mudgel, M., Awana, V.P.S., Kishan, H. and Bhalla, G.L. (2008) Significant Improvement of Flux Pinning and Irreversibility Field in Nano-Carbon-Doped $\mathrm{MgB}_{2}$ Superconductor. Solid State Communications, 146, 330-334. http://dx.doi.org/10.1016/j.ssc.2008.03.009

[9] Scanlan, R.M., Fietz, W.A. and Koch, E.F. (1975) Flux Pinning Centers in Superconducting Nb 3 Sn. Journal of Applied Physics, 46, 2244-2249. http://dx.doi.org/10.1063/1.321816

[10] Patnaik, S., Gurevich, A., Bu, S.D., Kaushik, S.D., Choi, J., Eom, C.B. and Larbalestier, D.C. (2004) Thermally Activated Current Transport in $\mathrm{MgB}_{2}$ Films. Physical Review B, 70, Article ID: 064503. http://dx.doi.org/10.1103/PhysRevB.70.064503

[11] Shekhar, C., Giri, R., Tiwari, R.S., Srivastavaa, O.N. and Malik, S.K. (2007) Enhancement of Flux Pinning and High Critical Current Density in Graphite Doped $\mathrm{MgB}_{2}$ Superconductor. Journal of Applied Physics, 102, Article ID: 093910. http://dx.doi.org/10.1063/1.2805650

[12] Ojha, N., Varma, G.D., Singh, H.K. and Awana, V.P.S. (2009) Effect of Rare-Earth Doping on the Superconducting Properties of $\mathrm{MgB}_{2}$. Journal of Applied Physics, 105, Article ID: 07E315.

[13] Zhou, S.H. and Dou, S.X. (2010) Properties of $\mathrm{MgB}_{2}$ Bulks after Combined Doping with Fe and C by Adding Iron(II) Lactate $\left(\mathrm{C}_{6} \mathrm{H}_{10} \mathrm{FeO}_{6}\right)$. Solid State Sciences, 12, 105-110. http://dx.doi.org/10.1016/j.solidstatesciences.2009.10.013

[14] Jiang, C.H. and Kumakura, H. (2007) Stoichiometry Dependence of the Critical Current Density in Pure and Nano-SiC Doped $\mathrm{MgB}_{2} /$ Fe Tapes. Physica C: Superconductivity, 451, 71-76. http://dx.doi.org/10.1016/j.physc.2006.11.001

[15] Dou, S.X., Pan, A.V., Zhou, S., Ionescu, M., Wang, X.L., Horvat, J., Liu, H.K. and Munroe, P.R. (2003) Superconductivity, Critical Current Density and Flux Pinning in $\mathrm{MgB}_{2-\mathrm{x}}(\mathrm{SiC})_{\mathrm{x} / 2}$ Superconductor after SiC Nanoparticle Doping. Journal of Applied Physics, 94, 1850. http://dx.doi.org/10.1063/1.1586467

[16] De Silva, K.S.B., Xu, X., Wang, X.L., Wexler, D., Attard, D., Xiang, F. and Dou, S.X. (2012) A Significant Improvement in the Superconducting Properties of $\mathrm{MgB}_{2}$ by Co-Doping with Graphene and Nano-SiC. Scripta Materialia, 67, 802-805. http://dx.doi.org/10.1016/j.scriptamat.2012.07.014

[17] De Silva, K.S.B., Xu, X., Gambhir, S., Wang, X.L., Li, W.X., Wallaceb, G.G. and Dou, S.X. (2011) Flux Pinning Mechanisms in Graphene-Doped $\mathrm{MgB}_{2}$ Superconductors. Scripta Materialia, 65, 634-637. http://dx.doi.org/10.1016/j.scriptamat.2011.06.047

[18] Tampieri, A., Celotti, G., Sprio, S. and Rinaldi, D. (2003) Effects of Cu and Other Metallic Dopings on the Supercon- 
ducting Properties of $\mathrm{MgB}_{2}$. International Journal of Modern Physics B, 17, 438-445. http://dx.doi.org/10.1142/S0217979203016078

[19] Ma, Z.Q., Liu, Y.C., Han, Y.J., Zhao, Q. and Gao, Z.M. (2008) Variation of the Enhancement Mechanism in the Critical Current Density of Cu-Doped $\mathrm{MgB}_{2}$ Samples Sintered at Different Temperatures. Journal of Applied Physics, 104, Article ID: 063917. http://dx.doi.org/10.1063/1.2982088

[20] Medvedeva, N.J., Ivanovskii, A.L., Medvedeva, J.E. and Freeman, A.J. (2001) Electronic Structure of $\mathrm{MgB}_{2}$ and Related Binary and Ternary Borides. Physical Review B, 64, Article ID: 205020. http://dx.doi.org/10.1103/PhysRevB.64.020502

[21] Tampieri, A., Celotti, G., Sprio, S., Rinaldi, D., Barucca, G. and Caciuffo, R. (2002) Effects of Copper Doping in $\mathrm{MgB}_{2}$ Superconductor. Solid State Communications, 121, 497-500. http://dx.doi.org/10.1016/S0038-1098(01)00514-2

[22] Chandrashekar, R.G., Tiwari, R.S., Srivastava, O.N. and Malik, S.K. (2007) Enhancement of Flux Pinning and High Critical Current Density in Graphite Doped $\mathrm{MgB}_{2}$ Superconductor. Journal of Applied Physics, 101, Article ID: 043906.

[23] Sprio, S., Rinaldi, D., Celotti, G., Pialorsi, E. and Tampieri, A. (2008) Structure and Superconducting Properties of Pure and Variously Doped Bulk $\mathrm{MgB}_{2}$ Obtained by Uniaxial and Isostatic Hot Pressing. Journal of Materials Science: Materials in Electronics, 19, 1012-1022. http://dx.doi.org/10.1007/s10854-007-9443-X

[24] Akamaru, S., Ishikawa, F., Nishimura, K., Abe, T. and Matsuyama, M. (2013) Effects of Metal (Ag, Sn and Zn) Nanoparticles Inserted into $\mathrm{MgB}_{2}$ Grain Boundaries on Transport and Superconducting Properties. Materials Transactions, 54, 2258-2264. http://dx.doi.org/10.2320/matertrans.M2013254

[25] Anderson, P.W. and Kim, Y.B. (1964) Hard Superconductivity: Theory of the Motion of Abrikosov Flux Lines. Reviews of Modern Physics, 36, 39-43. http://dx.doi.org/10.1103/revmodphys.36.39

[26] Zazo, M., Torres, L., Iniguez, J., de Francisco, C. and Munoz, J.M. (1984) Study of the Frequency and Low-Field Dependence of AC Susceptibility in YBaCuO. Journal of Applied Physics, 76, 7133-7135.

Anderson, P.W. (1962) Theory of Flux Creep in Hard Superconductors. Physical Review Letters, 9, 309-311. http://dx.doi.org/10.1103/PhysRevLett.9.309

[27] Qin, M.J., Wang, X.L., Soltanian, S., Li, A.H., Liu, H.K. and Dou, S.X. () Dependence of the Flux Creep Activation Energy on Current Density and Magnetic Field for the $\mathrm{MgB}_{2}$ Superconductor. Physical Review B, 64, Article ID: 060505.

[28] Ghigo, G., Botta, D., Chiodoni, A., Gerbaldo, R., Gozzelino, L., Laviano, F., Mezzetti, E. and Minetti, B. (2003) Pinning Energy and Vortex-Phase Diagram of $\mathrm{MgB}_{2}$ Bulk Materials. International Journal of Modern Physics B, 17, 584-589. http://dx.doi.org/10.1142/S0217979203016273

[29] Bean, C.P. (1962) Magnetization of Hard Superconductors. Physical Review Letters, 8, 250-253. http://dx.doi.org/10.1103/PhysRevLett.8.250

[30] Sidorenko, A., Zdravkov, V., Ryazanov, V., Horn, S., Klimm, S., Tidecks, R., Wixforth, A., Koch, T. and Schimmel, T. (2005) Thermally Assisted Flux Flow in $\mathrm{MgB}_{2}$ : Strong Magnetic Field Dependence of the Activation Energy. Phylosophical Magazine, 85, 1738-1790. http://dx.doi.org/10.1080/14786430500036678

[31] Jin, H., Wen, H.-H., Yang, H.-P., Liu, Z.-Y., Ren, Z.-A., Che, G.-C. and Zhao, Z.-X. (2003) Rigid Vortices in MgB 2. Applied Physics Letters, 83, 2626.

[32] Senatore, C., Polichetti, M., Zola, D., Di Matteo, T., Giunchi, G. and Pace, S. (2003) Vortex Dynamics and Pinning Properties Analysis of $\mathrm{MgB}_{2}$ Bulk Samples by AC Susceptibility Measurements. Superconductor Science and Technology, 16, 183-187. http://dx.doi.org/10.1088/0953-2048/16/2/310

[33] Patnaik, S., Gurevich, A., Bu, S.D., Kaushik, S.D., Choi, J., Eom, C.B. and Larbalestier, D.C. (2004) Thermally Activated Current Transport in $\mathrm{MgB}_{2}$ Films. Physical Review B, 70, Article ID: 064503.

http://dx.doi.org/10.1103/PhysRevB.70.064503 


\section{Appendix}

The quadruple $\left(U_{0}, T_{i r}, \alpha, \ln f_{0}\right)$ are to be considered as fitting parameters. The equation is forward sensitive to the $\alpha$ parameter and is better fitted using a backward optimisation algorithm with respect to this variable. Here again it is sufficient to set the value of $T_{i r r}$ to the onset temperature of third susceptibility harmonic valued at the higher frequency [28]—removing one of the unknowns, leaving the triplet $\left(U_{0}, \alpha, \ln f_{0}\right)$ to determine for every data line. Choosing a particular data line, the second and third parameters are determined through "standard" nonlinear fit, while $\alpha$ is seeded through a few preliminary tests (Equation (8) below indicates that in most cases the correct $\alpha<2$ ).

The value of the fitted $R^{2}$ coefficient is then computed for the fit and tabulated. The operation is then repeated for other values of $\alpha$ until a maximum $R^{2}$ case can be identified. That is taken to be the best fit for the data under consideration. There are sufficient data at every field and temperature value and this together with physical regularization permits a more than reasonable degree of confidence in this fitting system. In fact, for every line, $\{8,32,64,128,256\}$ respective $\alpha$ values are sampled to extract the best value. The answer always converges to a stable limit in every case.

Practically, there are two areas where problems can occur. The first is the intrinsic mathematical form. In practice the observed curvature, $C$, of the data points with respect to Equation (5) is small which implies that in Equation (6) one can perturb $\alpha=2+\delta$, and set:

$$
y=1-\left(\frac{T}{T_{i r r}}\right)^{2}
$$

and expand the curvature function as a series:

$$
C \approx(8+12 \delta) y^{\delta}
$$

For $T \approx T_{\text {irr }}$ and small $\delta<0$ the curvature: $C<0$ and varies directly as $\left[1-\left(T / T_{i r r}\right)^{2}\right]^{\delta}$ which is small and approximately constant (for a given curve) in this temperature range. This range is exactly where the measurements are assumed to be taken and the low curvature exhibited by the data sets in turn limit the range for $\alpha$ to small numbers. In fact a few numerical tests show that $\alpha \in[0.25,3.0]$ for every curve in the experiment.

These curves tend also to be a good guide to the reliability of the data itself. When for a data series the $\alpha$ values are closely grouped the data set shows a higher continuity and greater physical plausibility. If an $\alpha$

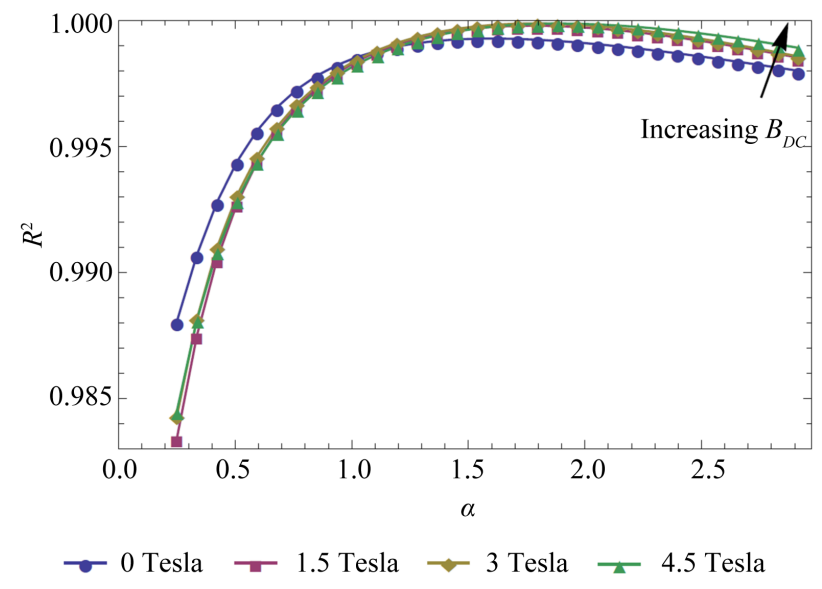

(a)

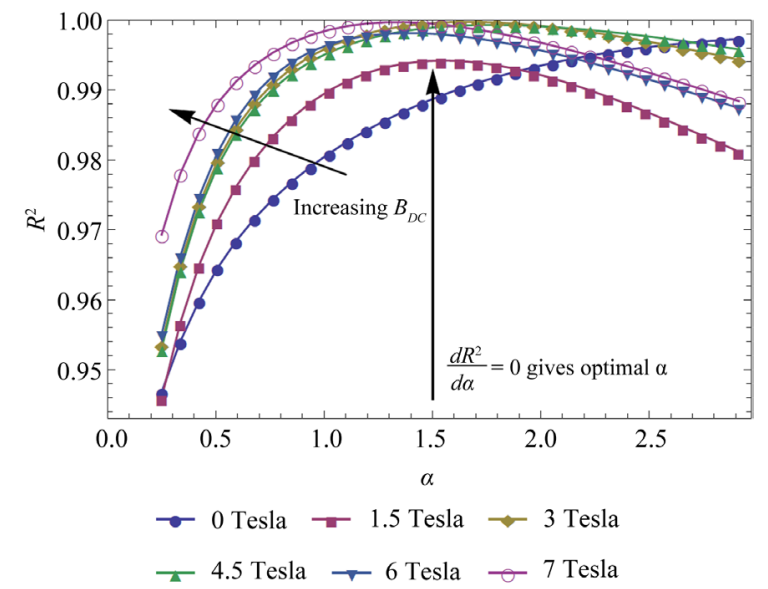

(b)

Figure 7. An example of the $\alpha$ parameter backward optimisation process: the nonlinear fit residual $R^{2}$ is maximised in every applied field case. (a) The $\alpha$ line grouping $\mathrm{MgB}_{2}$ doped with $\mathrm{Ag}$ at $20 \%$-note that the $\alpha$ grouping is close for every applied field case; (b) The $\alpha$ line grouping $\mathrm{MgB}_{2}$ doped with $\mathrm{Y}$ at $0.5 \%$ - the anomaly in the set is the $\alpha$ value to the far right, not surprising as it is the line for zero applied field at this $\mathrm{Y}$ doping percentage. 
value lies far outside the group then that data point would be an outlier and a value for $U_{0}$ developed from it might better be discarded or viewed at least with a certain degree of suspicion. In fact this situation happens for almost all the data series for the zero DC field plots (see Figure 7(b)).

Mathematically, that confirms the intuition that the three parameter nonlinear fit is a perturbation of Equation (5) in the irreversible temperature region and the relatively small values of $y$ and $\delta$ probably mean that it is not too far from a convex optimisation problem. This hypothesis is supported by Figure 7. In fact as already stated, working with the data using this model it is possible to obtain good fits with consistent values for all the parameters-if there are anomalies then they occur due to experimental issues as opposed to data analysis/extraction. 